\title{
Development and Validation of Chiral HPLC Method for Identification and Quantification of (R)-Enantiomer in Ezetimibe
}

\author{
Kameswara Rao Chimalakonda, Venkatanarayana Gudala, Madhusudhan Gutta, \\ Srinivasulu Polisetty, Sai Venkata Srinivas Koduri \\ Inogent Laboratory, Jawaharlal Nehru Technological University, Hyderabad, India \\ Email: Kameswararao_scientist@yahoo.co.in
}

Received May 6, 2012; revised May 26, 2012; accepted June 11, 2012

\begin{abstract}
A normal phase enantioselective high performance liquid chromatographic method was developed for enantiomeric resolution of Ezetimibe it reduces the overall delivery of cholesterol to the liver. The enantiomers of Ezetimibe were resolved on a Chiral Pak AS-H $(250 \times 4.6 \mathrm{~mm}, 5 \mu \mathrm{m})$ column using a mobile phase system containing n-hexane, ethanol, 2-propanol and trifloroacetic acid (84:12:4:0.1 v/v). The resolution between enantiomers was found to be more than 2.0. The limit of detection and limit of quantification of (R)-enantiomer were found to be $0.2 \mu \mathrm{g} / \mathrm{mL}$ and $0.5 \mu \mathrm{g} / \mathrm{mL}$, respectively, for $10 \mu \mathrm{L}$ injection volume. The sample solution and mobile phase were found to be stable for at least $48 \mathrm{~h}$. The final optimized method was successfully applied to separate (R)-enantiomer from Ezetimibe and was proven to be reproducible and accurate for the quantitative determination of $(\mathrm{R})$-enantiomer in bulk drugs.
\end{abstract}

Keywords: Ezetimibe; (R)-Enantiomer; Identification; Quantification and Validation

\section{Introduction}

Enantiomers of racemic drugs often show different behaviors in pharmacological action and metabolic process. It is not uncommon for one enantiomer to be active while other isomer is toxic in biological systems. The pharmaceutical industry has raised its emphasis on the generation of enantiomerically pure compounds before under taking phamarmacokinetic, metabolic, physiological, and toxicological evaluation in the search for drugs with grater therapeutic benefits and low toxicity [1,2]. Nowadays, chiral separations are playing more and more important role for the analysis of single enantiomers in the field of pharmaceutical industry [3]. However, the development of the methods for the quantitative analysis of Chiral compounds and for the assessment of enantiomeric purity is extremely challenging, because the same physical and chemical properties of the two enantiomers make discriminating and separating them very difficulty [4].

Ezetimibe was a selective inhibitor of intestinal cholesterol and related phytosterol absorption, is designated as 1-(4-fluorophenyl)-3(R)-[3-(4-fluorophenyl)-3(S)-hyd roxypropyl]-4(S)-(4-hydroxypenyl)-2-azetidinone [5]. Ezetimibe selectively prevents the absorption of cholesterol from dietary and biliary sources by blocking the transport of cholesterol through the intestinal wall. This reduces the overall delivery of cholesterol to the liver, there by promoting the synthesis of LDL receptors and the subsequent reduction in serum LDL-C [6,7]. Few HPLC methods for the determination of Ezetimibe and its pharmaceutical dosage forms were reported in literatures [810]. The (R)-enantiomer is an undesired enantiomer, which can be present as a chiral impurity without any pharmacological and toxicological reports by now. So it is essential to find a effective way to analyze the enantiomers of Ezetimibe, the chemical structures Ezetimibe and (R)enantiomer are shown in Figure 1.

Ezetimibe, is reported by Normal phase liquid chromatography using modified amylose as chiral stationary phases. The aim of this work was to optimize the chromatographic conditions in terms of temperature and mobile phase composition in order to separate, identify and quantify the enantiomer of Ezetimibe. The developed chiral HPLC method was reproducible and accurate for the quantitative determination of (R)-enantiomer in Ezetimibe.

\section{Experimental}

\subsection{Chemicals and Reagents}

Ezetimibe racemic mixture $( \pm)$ was obtained from the 


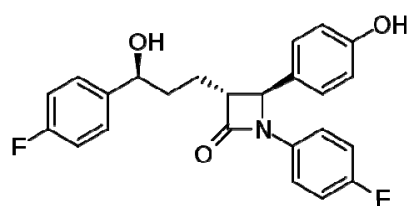

Figure 1. (3R,4S)-1-(4-fluorophenyl)-3-[(3S)-3-(4-fluorophenyl)-3-hydroxypropyl]-4-(4-hydroxyphenyl)azetidin-2-one.

R \& D department of Inogent Laboratory (Hyderabad, India). Chemical structure is presented in Figure 1. HPLC grade n-hexane, 2-propanal, trifluoroaceticacid and ethanol was purchased from Merck (Mumbai, India). Stock solution of analyte were prepared in n-hexane and ethanol in the ratio (80:20) (diluent) at a concentration 1 $\mathrm{mg} / \mathrm{mL}$. Ezetimibe and (R)-enantiomer (the undesired enantiomer (99\% ee)) were prepared in the laboratory by using preparative HPLC.

\subsection{Chromatographic Conditions}

Chromatography was carried out by using Agilent Technologies 1200 series instrument equipped with column oven, UV detector, and the data was processed using a computer program (chemestation, Dayton, $\mathrm{OH}$ ). The chromatographic conditions were optimized using a chiral stationary phase, Chiralpak AS-H column $(250 \times 4.6 \mathrm{~mm}$, $5 \mu \mathrm{m}$, Daicel, Japan). The isocratic mobile phase composition was a mixture of n-hexane, ethanol, 2-propanol and trifluoroacetic acid (84:12:4:0.1 v/v), which was pumped at a flow rate of $1 \mathrm{~mL} / \mathrm{min}$. The temperature of the column was maintained at $30^{\circ} \mathrm{C}$ and the eluent was monitored at a wavelength of $230 \mathrm{~nm}$. The injection volume was $10 \mu \mathrm{L}$.

The chromatographic parameters, including the retention factor $(\mathrm{k})$, the separation factor $(\alpha)$, and the resolution (Rs) were selected to evaluate the separation of compounds. All the chromatographic results were repeated three times.

\subsection{Sample Preparation}

Stock solutions of Ezetimibe $(1 \mathrm{mg} / \mathrm{mL})$ and (R)-enantiomer $(1 \mathrm{mg} / \mathrm{mL})$ were prepared by dissolving appropriate amount of the substance in n-hexane and ethanol in the ratio (80:20). The analyte concentration of Ezetimibe was fixed as $1000 \mu \mathrm{g} / \mathrm{mL}$. Ezetimibe solutions spiked with low levels of (R)-enantiomer were prepared by transferring calculated amount of undesired enantiomer stock solution with pipette into the calculated amount of Ezetimibe stock solution, and then the solution was added to volume with diluent and mixed well.

\subsection{Validation of the Method}

The specificity of the method is performed by injecting both isomers and recemic mixture individually. The spe- cificity determined by using peak purity, resolution.

The system suitability of the method performed by adding known concentration $(5.0 \mu \mathrm{g} / \mathrm{mL})$ of undesired isomer to Ezetimibe. The system suitability is confirmed by using resolution, tailing factor, Tangent.

Method reproducibility was determined by measuring repeatability and intermediate precision of retention times and peak areas for each enantiomer. The repeatability of the method was determined by analyzing six replicate injections containing Ezetimibe $(1000 \mu \mathrm{g} / \mathrm{mL})$ spiked with (R)-enantiomer $(0.3 \%, 3 \mu \mathrm{g} / \mathrm{mL})$. The intermediate precision was determined over 3 days by performing six successive injections $(n=6)$ each day and also performed intermediate precision with different system, different analyst and with different column by using six injections $(n=6)$.

The limit of detection (LOD) and limit of Quantification (LOQ) for (R)-enantiomer was achieved by injecting a series of dilute solutions of by using standard deviation slope method (ICH Q2 (R1)). The LOQ level precision of the developed chiral method for (R)-enantiomer was checked by analyzing six solutions of (R)-enantiomer prepared at LOQ level and calculating the percentage relative standard deviation of area.

Detector response linearity was assessed by preparing eight calibration sample solutions of (R)-enantiomer covering from $0.5 \mu \mathrm{g} / \mathrm{mL}$ (LOQ) to $12 \mu \mathrm{g} / \mathrm{mL}(0.5,1.5,3.0$, 4.5, 7.5, 9.0, 10.5 and $12 \mu \mathrm{g} / \mathrm{mL}$ ) in diluent. The regression curve was obtained by plotting peak area versus concentrations, using the least square method. The percentage relative standard deviation of the slope and yintercept of the calibration curve was calculated.

The accuracy of method was carried out by injecting known concentration of R-enantiomer to the Ezetimibe. The accuracy was calculated in terms of recovery (\%). The study was carried out in triplicate at covering from $1.5 \mu \mathrm{g} / \mathrm{mL}$ to $12 \mu \mathrm{g} / \mathrm{mL}(1.5,3.0,4.5,7.5,9.0,10.5$ and $12 \mu \mathrm{g} / \mathrm{mL}$ ) in diluent. The recovery of (R)-enantiomer was calculated.

To determine robustness of the method, flow rate was changed at the pace of 0.2 units from 0.8 to $1.2 \mathrm{~mL} / \mathrm{min}$. The effect of change in the percent 2-propanol and ethanol $( \pm 10 \%)$, and column temperature at $25^{\circ} \mathrm{C}$ and $35^{\circ} \mathrm{C}$ instead of $30^{\circ} \mathrm{C}$ were studied, and the other chromatographic conditions were held constant as stated previously.

The solution stability of Ezetimibe at analyte concentration was studied by keeping the solution in tightly capped volumetric flask at room temperature on a laboratory bench for $48 \mathrm{~h}$. The content of (R)-enantiomer was checked at $6 \mathrm{~h}$ interval up to the study period. Mobile phase stability was carried out by evaluating the content of (R)-enantiomer in Ezetimibe sample solutions prepared freshly at $6 \mathrm{~h}$ interval of $48 \mathrm{~h}$. The same mobile 
phase was used during the study period.

\section{Results and Discussion}

\subsection{Optimization of Chromatographic Conditions}

Racemic mixture solution of Ezetimibe and (R)-enantiomer $(1000 \mu \mathrm{g} / \mathrm{mL}$ each) prepared in diluents was used in the method development. To develop a rugged and suitable normal phase HPLC method for the separation of the two enantiomers, different stationary phases and mobile phases were employed. Chiralpak AS-H column $(250 \times 4.6 \mathrm{~mm}, 5 \mu \mathrm{m})$ with mobile phase consisting of $\mathrm{n}$ hexane, ethanol, 2-propanol and trifluoroacetic acid (84: $12: 4: 0.1 \mathrm{v} / \mathrm{v}$ ) was used. It was continued to select the best stationary and mobile phases that would give optimum resolution and selectivity for the two enantiomers. There was an indication of separation on Chiralpak AS-H (250 $\times 4.6 \mathrm{~mm}, 5 \mu \mathrm{m}$ ) column using a mobile phase consisting of n-hexane, ethanol, 2-propanol and trifluoroacetic acid acid (84:12:4:0.1 v/v). The composition of the mobile phase was optimized to enhance the chromatographic efficiency and resolution between the enantiomers. The results of resolution factor (Rs) and selectiveity factor $(\alpha)$ are summarized in Table 1. Based on the data obtained from the method development and optimization activities, Chiralpak AS-H $(250 \times 4.6 \mathrm{~mm}, 5 \mu \mathrm{m})$ column with mobile phase of n-hexane, ethanol, 2-propanol and trifluoroacetic acid (84:12:4:0.1 v/v) was selected from the method development. The flow rate of the final method was $1.0 \mathrm{~mL} / \mathrm{min}$ with injection volume $10 \mu \mathrm{L}$. The column temperature was $30^{\circ} \mathrm{C}$, and the detection wavelength was $230 \mathrm{~nm}$. Under these conditions, the two enantiomers were separated well and the peak of (R)-enantiomer eluted before the peak of Ezetimibe. In the optimized method, the typical retention time of (R)-enantiomer and Ezetimibe were 10.4, $12.2 \mathrm{~min}$, respectively. Base line separation of Ezetimibe and (R)-enantiomer was obtained with total run time of $25 \mathrm{~min}$. The separation of an approximately $1: 1(\mathrm{wt} / \mathrm{wt})$ mixture solution (in diluent) of the two enantiomers in shown in Figure 2 HPLC chromatogram of Ezetimibe bulk drug sample $(1000 \mu \mathrm{g} / \mathrm{mL})$ spiked with (R)-enantiomer (0.3\%) was shown in Figure 3.

\subsection{Validation Results of the Method}

The HPLC condition of the final method was evaluated for its specificity, LOD, LOQ, linearity, accuracy, precision, robustness and stability.

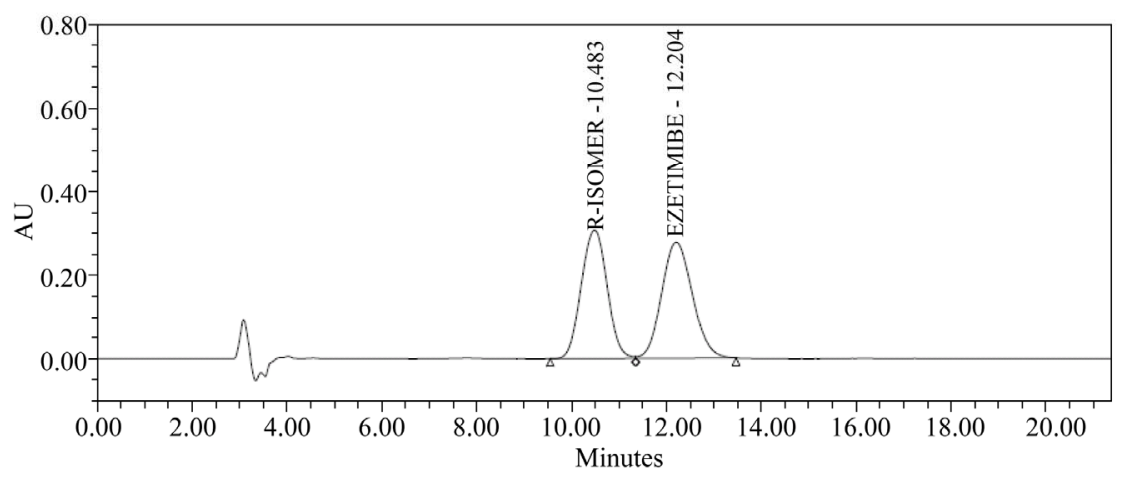

Figure 2. R-Isomer and Ezetimibe (1:1).

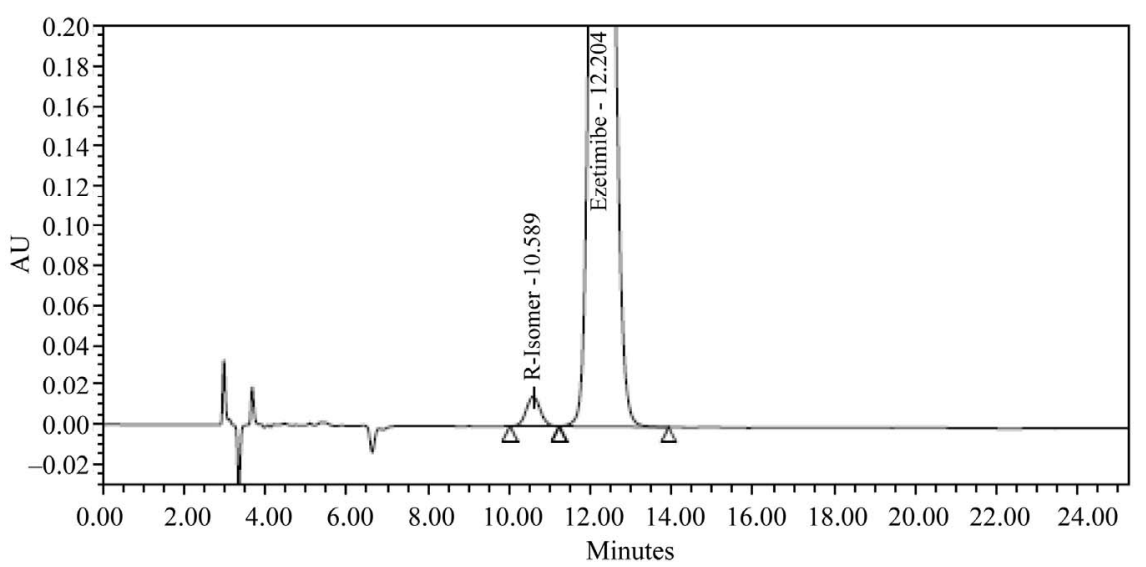

Figure 3. Ezetimibe $+0.3 \%$ R-Isomer spiked. 
Table 1. Specificity.

\begin{tabular}{cccc}
\hline Compound & Purity angle & Purity threshold & Peak purity \\
\hline R-Isomer & 0.9325 & 2.3458 & Pass \\
Ezetimibe & 3.5871 & 4.6204 & Pass \\
\hline
\end{tabular}

The specificity of the method was determined by using peak purity.

The specificity results are given in Table 1 .

The LOD and LOQ concentrations were estimated 0.2 $\mu \mathrm{g} / \mathrm{mL}$ and $1.0 \mu \mathrm{g} / \mathrm{mL}$ for (R)-enantiomer. Calculated the LOD and LOQ by using standard and slope method. The LOD and LOQ values for (R)-enantiomer were $0.2 \mu \mathrm{g}$ and $0.5 \mu \mathrm{g}$. Method precision for (R)-enantiomer at LOQ was less than $2.0 \%$ RSD. Therefore, this method had adequate sensitivity for the detection and estimation of (R)-enantiomer in Ezetimibe. R-Isomer and Ezetimibe individual sample chromatograms are shown in Figures 4 and 5.

Good linearity of (R)-enantiomer was evaluated over eight levels of (R)-enantiomer solutions from $0.5 \mu \mathrm{g} / \mathrm{mL}$ to $12 \mu \mathrm{g} / \mathrm{mL}$, with the linear regression equation $\mathrm{y}=\mathrm{mx}$ $+\mathrm{c}$, where $\mathrm{x}$ is the concentration in $\mu \mathrm{g} / \mathrm{mL}$, and $\mathrm{y}$ is the corresponding peak area of undesired enantiomer in $\mathrm{mV} / \mathrm{s}$. We observed linear results with respect to concentration for (R)-enantiomer. The correlation coefficient value is more than 0.999 (Table 2). The linearity graph was shown in Figure 6.

The standard addition and recovery experiments were conducted for (R)-enantiomer in bulk samples in triplicate at $1.5 \mu \mathrm{g} / \mathrm{mL}$ to $12 \mu \mathrm{g} / \mathrm{mL}(1.5,3.0,4.5,7.5,9.0$, 10.5 and $12 \mu \mathrm{g} / \mathrm{mL})$. The accuracy was in terms of recovery (\%). The recovery was calculated by back calculated concentration at each level in each preparation. The recovery is not less than $99.6 \%$ and not more than 102.2\% (Table 3).

The repeatability and intermediate precision were expressed as relative standard deviation (RSD). For this study, solution of Ezetimibe $(1000 \mu \mathrm{g} / \mathrm{mL})$ spiked with (R)-enantiomer $(0.3 \%, 3 \mu \mathrm{g} / \mathrm{mL})$ was analyzed in six injections to establish repeatability. RSD values were better than $0.5 \%$ for the retention times of both the enantiomers. In the intermediate precision study results shown that RSD values were in same order of magnitude than those obtained for repeatability studies (Table 4). All these values indicated that the method was precise.

The method robustness studies were demonstrated by adjusting flow rate, column temperature and mobile phase composition variations. The chromatographic resolution of Ezetimibe and (R)-enantiomer was more than 2.0 under all separation conditions tested (Table 5), demonstrated sufficient robustness.

The stability of the solution and mobile phase used in
Table 2. Linearity.

\begin{tabular}{ccc}
\hline S.No & Concentration (\%) & R-Isomer peak (n = 6) \\
\hline 1 & 0.05 & 11331.0 \\
2 & 0.15 & 33375.3 \\
3 & 0.30 & 68981.3 \\
4 & 0.45 & 101922.7 \\
5 & 0.75 & 168752.7 \\
6 & 0.90 & 202998.7 \\
7 & 1.05 & 237024.7 \\
8 & 1.20 & 270043.7 \\
Correlation coefficient & & 0.99998 \\
Slope & & 225064.81 \\
Y-intercept & & 358.22 \\
$\mathbf{r}^{2}$ & & 0.99996 \\
\hline
\end{tabular}

Table 3. Accuracy.

\begin{tabular}{ccc}
\hline Added amount $(\boldsymbol{\mu g})$ & Recovery (\%) & \%RSD (n = 3) \\
\hline 1.5 & 99.33 & 1.01 \\
3.0 & 101.67 & 0.76 \\
4.5 & 102.00 & 0.33 \\
7.5 & 101.87 & 0.26 \\
9.0 & 100.33 & 0.68 \\
10.5 & 99.24 & 1.13 \\
12.0 & 100.75 & 0.25 \\
\hline
\end{tabular}

Table 4. Ruggedness.

\begin{tabular}{cc}
\hline Name & \%RSD (n = 6) \\
\hline Day-1 & 0.2 \\
Day-2 & 0.5 \\
Day-3 & 0.4 \\
Diff system & 0.6 \\
Diff column & 0.7 \\
Diff analyst & 0.4 \\
\hline
\end{tabular}

Table 5. Robustness.

\begin{tabular}{lccc}
\hline Description & USP Tailing & $\begin{array}{c}\text { USP } \\
\text { Tangent }\end{array}$ & $\begin{array}{c}\text { USP } \\
\text { Resolution }\end{array}$ \\
\hline Column flow: $0.80 \mathrm{~mL} / \mathrm{min}$ & 1.1 & 3817 & 2.5 \\
Column flow: $1.20 \mathrm{~mL} / \mathrm{min}$ & 1.0 & 4525 & 2.1 \\
Column Temp: $25^{\circ} \mathrm{C}$ & 1.0 & 4049 & 2.1 \\
Column Temp: $35^{\circ} \mathrm{C}$ & 1.1 & 3302 & 2.4 \\
Organic ratio: $110 \%$ & 1.1 & 3142 & 2.1 \\
Organic ratio: $90 \%$ & 1.1 & 4557 & 2.4 \\
\hline
\end{tabular}




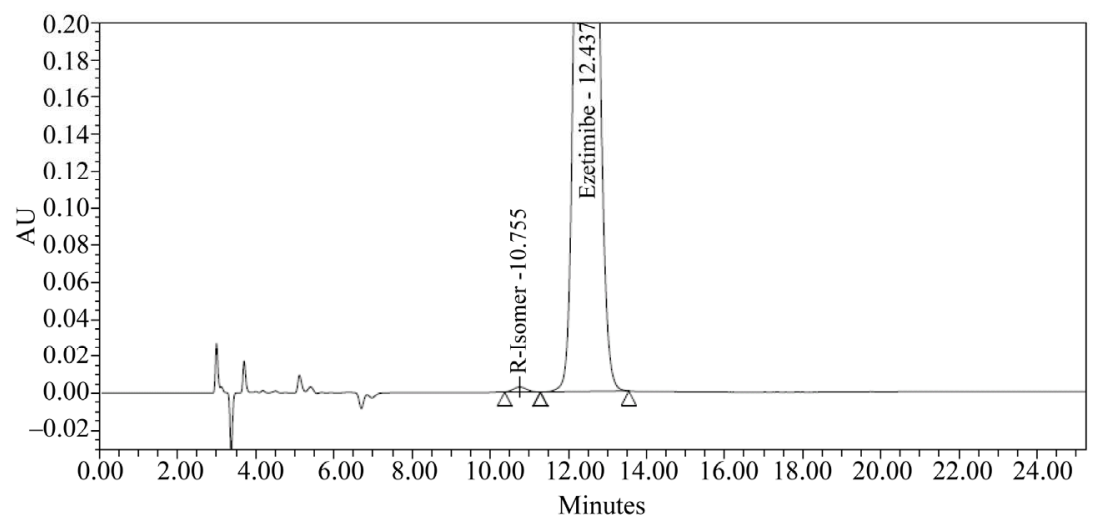

Figure 4. Ezetimibe sample as such.

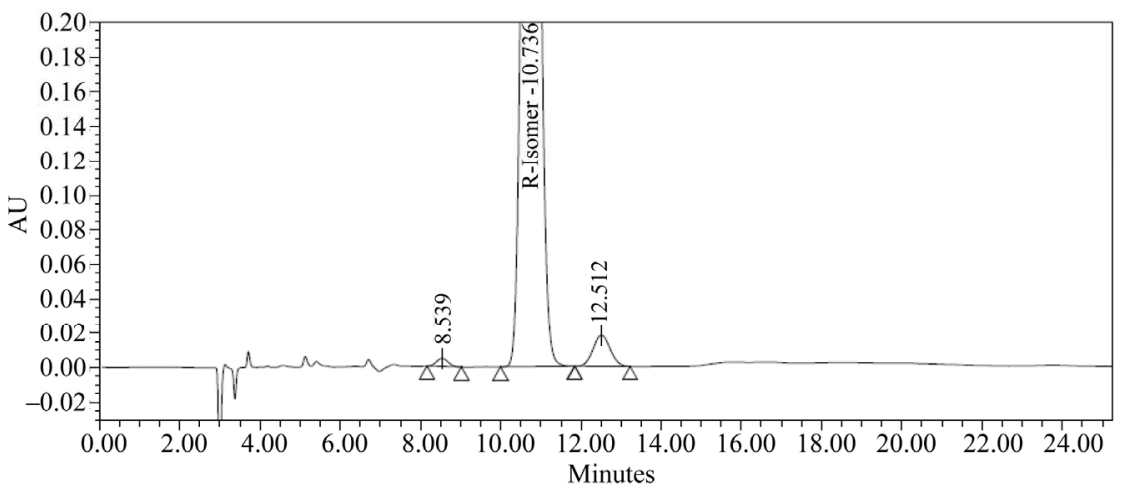

Figure 5. Chromatogram of R-Isomer.

R-Isomer linearity from $0.05 \%$ (LOQ) to $1.20 \%$

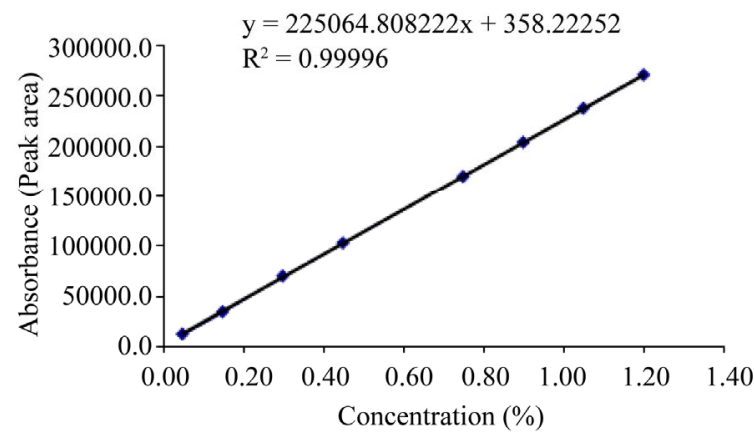

Figure 6. Linearity graph.

this method was tested over a long time. No significance change in (R)-enantiomer content was observed in Ezetimibe sample during solution stability and mobile phase stability experiments, and the RSD values were less than $2.0 \%$ for (R)-enantiomer peak area. No unknown peak was observed in above stability conditions. Hence, the Ezetimibe sample solution and the mobile phase were stable for at least $48 \mathrm{~h}$.

\section{Conclusion}

A simple, specific, linear, accurate and precise normal phase HPLC method was successfully developed, which was capable of separating the undesired enantiomer from Ezetimibe. Amylose based chiral column Chiralpak AS$\mathrm{H}$ column was found to be selective for the enantiomers of Ezetimibe. The developed and validated method can be used for the chiral purity testing of Ezetimibe. The developed method is also stable and can be used for the quantitative determination of chiral impurity in bulk materials.

\section{Batch Analysis}

By using this method we can anlyze and quantify (R)enantiomer in Ezetimibe in manufacturing batches and $\mathrm{R}$ \& D samples. We get repeatable results in all QC sample.

\section{Acknowledgements}

The authors would like to acknowledge the management of Inogent Laboratories Private Limited, Hyderabad, India.

\section{REFERENCES}

[1] H. Caner, E. Groner, L. Levy and I. Agranat, "Trends in the Development of Chiral Drugs,” Drug Discovery To- 
day, Vol. 9, No. 3, 2004, pp. 105-110. doi:10.1016/S1359-6446(03)02904-0

[2] J. Caldwell, "Importance of Stereospecific Bionalytical Monitoring in Drug Development," Journal of Chromatography A, Vol. 719, No. 1, 1996, pp. 3-13. doi:10.1016/0021-9673(95)00465-3

[3] N. M. Maier, P. Franco and W. Lindner, "Separation of Enantiomers: Needs Challenges, Perspectives,” Journal of Chromatography A, Vol. 906, No. 1-2, 2001, pp. 3-33. doi:10.1016/S0021-9673(00)00532-X

[4] T. E. Beesley and R. P. W. Scott, "Chiral Chromatography,” John Wiley \& Sons, Ltd., Chichester, 1998, pp. 2326.

[5] X. X. Xu, R. Fu, S. W. Chen, et al., "Ezetimibe Analogs with a Reorganized Azetidinone Ring: Design, Synthesis, and Evaluation of Cholesterol Absorption Inhibitions," Bioorganic \& Medicinal Chemistry Letter, Vol. 17, No. 1, 2007, pp. 101-104. doi:10.1016/j.bmcl.2006.09.078

[6] E. Leitersdorf, "Selective Cholesterol Absorption Inhibition: A Novel Strategy in Lipid-Lowering Management," International Journal of Clinical Practice, Vol. 56, No. 2, 2002, pp. 116-119.
[7] M. Van Heek, C. Farley and D. S. Compton, "Ezetimibe Selectively Inhibits Intestinal Cholesterol Absorption in Rodents in Presence and Absence of Exocrine Pancreatic Function," British Journal of Pharmacology, Vol. 134, No. 2, 2001, pp. 409-417. doi:10.1038/sj.bjp.0704260

[8] S. Singh, B. Singh, R. Bahuguna, L. Wadhwa and R. Saxena, "Stress Degradation Studies on Ezetimibe and Development of a Validated Stability-Indicating HPLC Assay," Journal of Pharmaceutical and Biomedical Analysis, Vol. 41, No. 3, 2006, pp. 1037-1040. doi:10.1016/j.jpba.2006.01.030

[9] R. Sistla, V. S. Tata, Y. V. Kashyap, D. Chandrasekar and P. V. Diwan, "Development and Validation of a ReversedPhase HPLC Method for the Determination of Ezetimibe in Pharmaceutical Dosage Forms,” Journal of Pharmaceutical and Biomedical Analysis, Vol. 39, No. 3-4, 2005, pp. 517-522. doi:10.1016/j.jpba.2005.04.026

[10] B. G. Chaudhari, N. M. Patel and P. B. Shah, "Stability-Indicating Reversed-Phase Liquid Chromatographic Method for Simultaneous Determination of Simvastatin and Ezetimibe from Their Combination Drug Products,” Journal of AOAC International, Vol. 90, No. 5, 2007, pp. 1242-1249. 